\title{
DIAGONAL: A numerical solution of the Stokes transfer equation
}

\author{
A. López Ariste and M. Semel \\ DASOP, Observatoire de Paris, Section de Meudon, 92195 Meudon principal Cedex, France
}

Received April 28; accepted July 7, 1999

\begin{abstract}
In a previous paper (Semel \& López Ariste 1999, hereafter referred to as Paper I) the authors found a new solution for the integration of the radiative transfer equation for polarized light (RTE). The present paper is a continuation of that work, and shows how this solution has been incorporated into a new numerical code for the integration of the RTE. This code, called DIAGONAL, is characterized by an analytical handling of most model atmospheres, differing in that from other codes. It gives exact solutions in new, non-trivial cases. In the general case when no complete analytical solution is available, the code resorts to a multi-layer technique, thus providing a solution that combines an approximated analytical model and a minimized residual treated linearly. The algorithm is shown both to be very stable and to reach a high precision even for a small number of integration layers. This results in a very fast code. A comparison with DELO has been carried out. DIAGONAL is available, upon request to the authors.
\end{abstract}

Key words: methods: numerical — Sun: atmospheres

\section{Introduction}

The radiative transfer equation for polarized light (RTE) has resisted all the approaches for a practical general solution even in those cases in which the source function is well determined (Landi Degl'Innocenti \& Landi Degl'Innocenti 1985). In the familiar scalar case when polarization is not taken into account, the solution of the RTE is straightforward, provided that the source function is given as a function of the integration variable (i.e. optical or geometrical depth). This is not the case, however, for polarized light. Going from one to four dimensions introduces new and fundamental problems. Paper I demonstrated that the

Send offprint requests to: A. López Ariste

Correspondence to: Arturo.Lopez@obspm.fr non-commutativity properties of a general absorption matrix are at the origin of all those difficulties, and proposed a way of dealing with them. The insights gained from this work have indeed suggested a method to obtain a general analytical solution not based on infinite series (Magnus 1954; Landi Degl'Innocenti 1987). This solution has been presented by López Ariste \& Semel (1999) but further work will be necessary before a numerical code based on it can be implemented. On the other hand, the solution found in Paper I for the case of commuting absorption matrices proved to be very powerful, and at the same time very easy to implement into a numerical code. The set of commuting matrices includes all the cases for which a particular solution is already known (Unno-Rachkowsky solution - Rachkowsky 1967-, constant matrix case -Landi Degl'Innocenti \& Landi Degl'Innocenti 1985-, ...) as well as some new non-trivial cases, for instance those in which the azimuth of magnetic field varies linearly with depth. In order to extend this approach to more general absorption matrices, including non-commuting ones, it was proposed in Paper I to firstly approximate any absorption matrix by a commuting one, for which a solution was known, and secondly to improve this solution by linearizing the residual. In this paper we will present the numerical code that implements that algorithm, which we have called DIAGONAL, and will study its performances using DELO (Rees et al. 1989) as a benchmark.

When introducing a new numerical code, analysis of errors and convergence rates is very important. In relation to RTE, this analysis has once again been hindered by the absence of an adequate analytical solution. Comparison with Rachkowsky solution (Rachkowsky 1967) in a MilneEddington atmosphere with constant absorption matrix, used to serve as a first indication of how good an algorithm was or how it converged (Rees et al. 1989; Bellot Rubio et al. 1998). This is however not a conclusive test because, due to its linear source function, any integration method more accurate than first order in the integration variable (usually $z$ the geometrical light path, or $\tau$ the optical depth) will give an exact solution for 
these kinds of atmosphere models. Although other solutions with non-linear source functions (Lites et al. 1988) or non-constant absorption matrices (Landi Degl'Innocenti \& Landi Degl'Innocenti 1985) are known, they have apparently never been used for this purpose. Therefore performances have been usually calculated by comparison to previous algorithms, and so we shall do the same with DIAGONAL. The basic assumption under this comparison test is that any accepted algorithm gives the exact solution (up to a given numerical precision) for a large number of integration layers. This assumption is revealed to be perfectly well founded. Nevertheless, the study of the algorithms when compared to an analytical solution for non-trivial cases seems to be very desirable. The algorithm proposed in Paper I provides an exact solution for some special non-trivial cases and, once the numerical code is shown to perform correctly, this fact can be used to test other numerical algorithms.

In the same line of thought we will make use in Sect. 2 of the formal solution given by Magnus (1954, see also the Appendix of Paper I for a resumée of this seminal paper) to characterize the algorithm in terms of convergence and errors. In Sect. 3 we will show in detail the way in which the proposed algorithm has been implemented. Finally Sect. 4 will be dedicated to show some numerical comparisons of the new code, called DIAGONAL, with the well-known DELO, and, as proposed above, of the convergence of DELO to the correct solution given by DIAGONAL in particular (but not trivial) cases.

\section{Error analysis}

Usually (e.g. Paper I, López Ariste \& Semel 1999 or the original paper by Landi Degl'Innocenti \& Landi Degl'Innocenti 1985), the formal solution to the RTE is presented as

$\boldsymbol{I}(z)=\int_{z_{0}}^{z} \mathrm{O}\left(z, z^{\prime}\right) \boldsymbol{J}\left(z^{\prime}\right) \mathrm{d} z^{\prime}+\mathrm{O}\left(z, z_{0}\right) \boldsymbol{I}\left(z_{0}\right)$,

where $\boldsymbol{I}$ is the Stokes vector, $\boldsymbol{J}$ the emission vector, $z$ the light path through the atmosphere and $\mathrm{O}\left(z, z_{0}\right)$ the so-called evolution operator. This expression can be seen as the particular solution of an inhomogeneous first-order differential equation whose homogeneous counterpart has a solution given by the evolution operator:

$\frac{\mathrm{d}}{\mathrm{d} t} \mathrm{O}\left(t, z_{0}\right)=-\mathrm{K}(t) \mathrm{O}\left(t, z_{0}\right)$,

where $\mathrm{K}$ denotes the absorption matrix and where

$\mathrm{O}\left(z_{0}, z_{0}\right)=1$

the $4 \times 4$ identity matrix. Magnus (1954) provides us with a general solution to this kind of equation in the form of a unique exponential:

$\mathrm{O}\left(z, z_{0}\right)=\mathrm{e}^{-\Omega\left(z, z_{0}\right)}$.
The exponent $\Omega\left(z, z_{0}\right)$ is given as an infinite series whose 2 first terms are

$\Omega\left(z, z_{0}\right)=\int_{z_{0}}^{z} \mathrm{~K}(t) \mathrm{d} t+\frac{1}{2} \int_{z_{0}}^{z}\left[\mathrm{~K}(t), \int_{z_{0}}^{t} \mathrm{~K}(\sigma) d \sigma\right] \mathrm{d} t+\ldots$

The rest of the terms in the series are higher recursive commutators between the absorption matrix and its integral. This solution, impractical from a numerical point of view, will allow us nevertheless to describe and clarify the basic sources of numerical errors when integrating the RTE. Introducing Eq. (3) in Eq. (1) we obtain:

$\boldsymbol{I}(z)=\int_{z_{0}}^{z} \mathrm{e}^{-\Omega\left(z, z^{\prime}\right)} \boldsymbol{J}\left(z^{\prime}\right) \mathrm{d} z^{\prime}+\mathrm{e}^{-\Omega\left(z, z_{0}\right)} \boldsymbol{I}\left(z_{0}\right)$.

Any numerical integration algorithm must solve, implicitly or explicitly, three problems:

1. Determination of the exponent $\Omega\left(z, z^{\prime}\right)$.

2. Calculation of the exponential.

3 . Integration of the emission vector.

Below we shall discuss these three issues in greater detail.

\subsection{Determination of the exponent $\Omega\left(z, z^{\prime}\right)$}

The only known attempt to explicitly calculate the second or higher terms in Eq. (3) was done by López Ariste \& Semel (1998) for a matrix linear with the integration variable. It was given for illustrative purposes and reflects the difficulties of this computation in the form given by Magnus. The analytical solution given by López Ariste \& Semel (1999) gives some hope as to the inclusion of the commutators of the absorption matrix with its integral in the solution, but some further work is still necessary. From the numerical codes side, it is usually difficult to disentangle the determination of $\Omega\left(z, z^{\prime}\right)$ from the problem of calculation of the exponential. For instance, DELO integrates the diagonal of the absorption matrix and linearizes all the rest. This linear approximation should take into account the calculation of the exponential of the offdiagonal terms as well as the higher terms of $\Omega\left(z, z^{\prime}\right)$. In practice, DELO imposes a constant matrix for each layer, the variations with depth of the model atmosphere being taken into account by the computation of numerous layers. For a constant matrix, all the commutators are null and $\Omega\left(z, z^{\prime}\right)$ becomes very easy to calculate. The Canarias Hermite method (Bellot Rubio et al. 1998; Ruiz Cobo et al. 1998) uses a completely different reasoning: instead of worrying about the formal solution of the RTE, this method never integrates explicitly the equation but takes into account the derivatives of the solution, which can be obtained directly from the differential equation. In that sense this method implicitly calculates the whole Magnus' series, as it is built such as to ensure that its derivatives satisfy always the RTE. On the other hand, the number of derivatives which can be calculated and the number of points at which they can be calculated is limited. The result is equivalent to a sampling of the solution, with the 


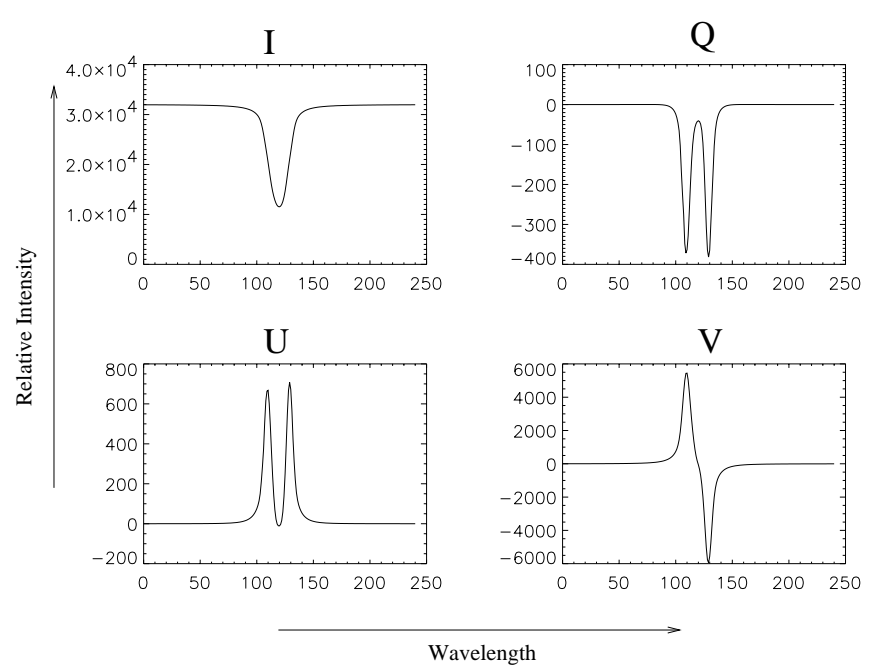

Fig. 1. Stokes profiles for the $6301 \AA$ Fe I line in a model with magnetic field equal to $2300+800 \log \tau \mathrm{G}$, inclination equal to $45^{\circ}+10 \log \tau$, velocity in the l.o.s. equal to $0.3 \log \tau \mathrm{km} \mathrm{s}^{-1}$, constant azimuth equal to $40^{\circ}$ and ratio of the line to continuum absorption coefficient equal to 40

usual associated errors of any sampling. Perhaps the most important among them are the errors associated with the calculation of the exponential (see next subsection) and the problem of the oscillating evolution operator (Bellot Rubio et al. 1998), which will be briefly discussed below.

The solution proposed in Paper I, and implemented in DIAGONAL, integrates explicitly the RTE. The absorption matrix is separated into two pieces: one $\left(\mathrm{K}_{\mathrm{o}}\right)$ which commutes with its own integral and another which does not commute. For the first one $\Omega\left(z, z^{\prime}\right)$ is immediately determined from the first term of the Magnus' series:

$\Omega_{\mathrm{o}}\left(z, z^{\prime}\right)=\int_{z}^{z^{\prime}} \mathrm{K}_{\mathrm{o}}(t) \mathrm{d} t$.

The difference with the constant-matrix solution is that Paper I provides the necessary and sufficient conditions for any absorption matrix to commute. DIAGONAL takes advantage of that and can, for instance, incorporate some gradients into the commuting part. The best example is the one of a linear variation with optical depth of the azimuth of magnetic field (see Paper I).

For the residual part we made a choice and we linearize it in a similar way to DELO with the off-diagonal terms. The residual is very small compared to the whole, so that this linearization is a fair approximation. Furthermore, the atmosphere is divided therefore improving the linear approximation and diminishing the error. It is important to remark that the number of layers necessary to keep under control errors is very small compared to DELO, as most of the problem is solved analytically through $\Omega_{\mathrm{o}}$.

The choice of a DELO-like linear approximation is not the sole one available and even not the best. The residual part obeys Eq. (66) of Paper I and a variety of numerical methods can be thought of to solve it. Our choice responds
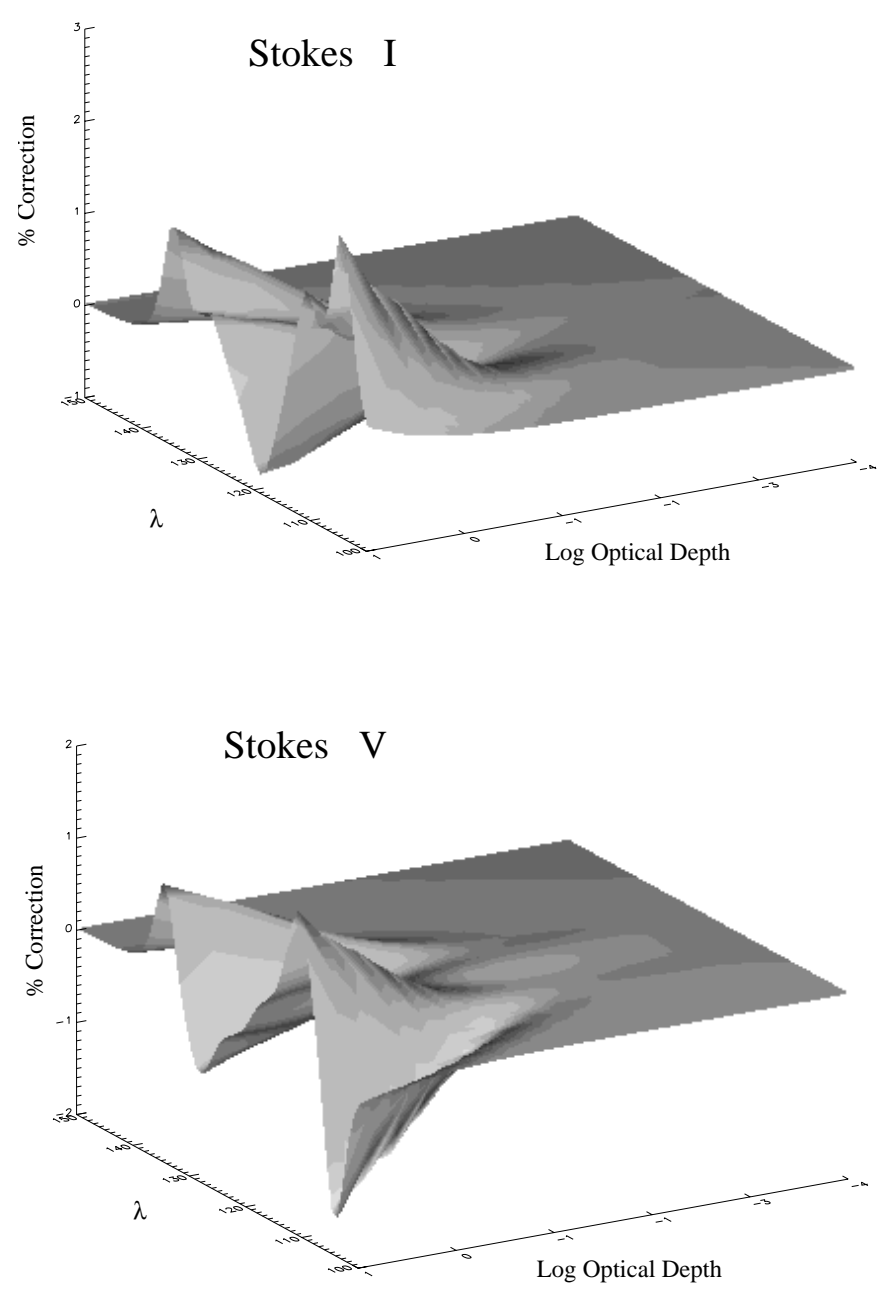

Fig. 2. Linear correction in percent of the total signal applied to $I$ and $V$ of Fig. 1 with 15 integration layers

to simplicity. As we shall see later, the transformations involved in the analytical part of the solution put a price in terms of computation time per layer. Further sophistications in solving the residual risked increasing this time and diminishing the performance of the code.

How good is the hypothesis that the residual is really a residual, i.e., small compared with the analytical part? To answer this question we have drawn the residual in terms of percentage of the final signal at each wavelength calculated and at each node in depth, for a model with strong gradients in intensity and azimuth of magnetic field and in velocity in the l.o.s. (see the next section for a full characterization of the model atmosphere used in this and other tests presented in this paper). The Stokes profiles produced by this model atmosphere for the Fe I $6301 \AA$ line are presented in Fig. 1. Figures 2 and 3 show the residual when the model is integrated using 15 layers. In view of them, both the name residual applied to the non-commuting part of the initial absorption matrix and the linear approximation applied to it are completely justified. Of course this residual correction becomes 

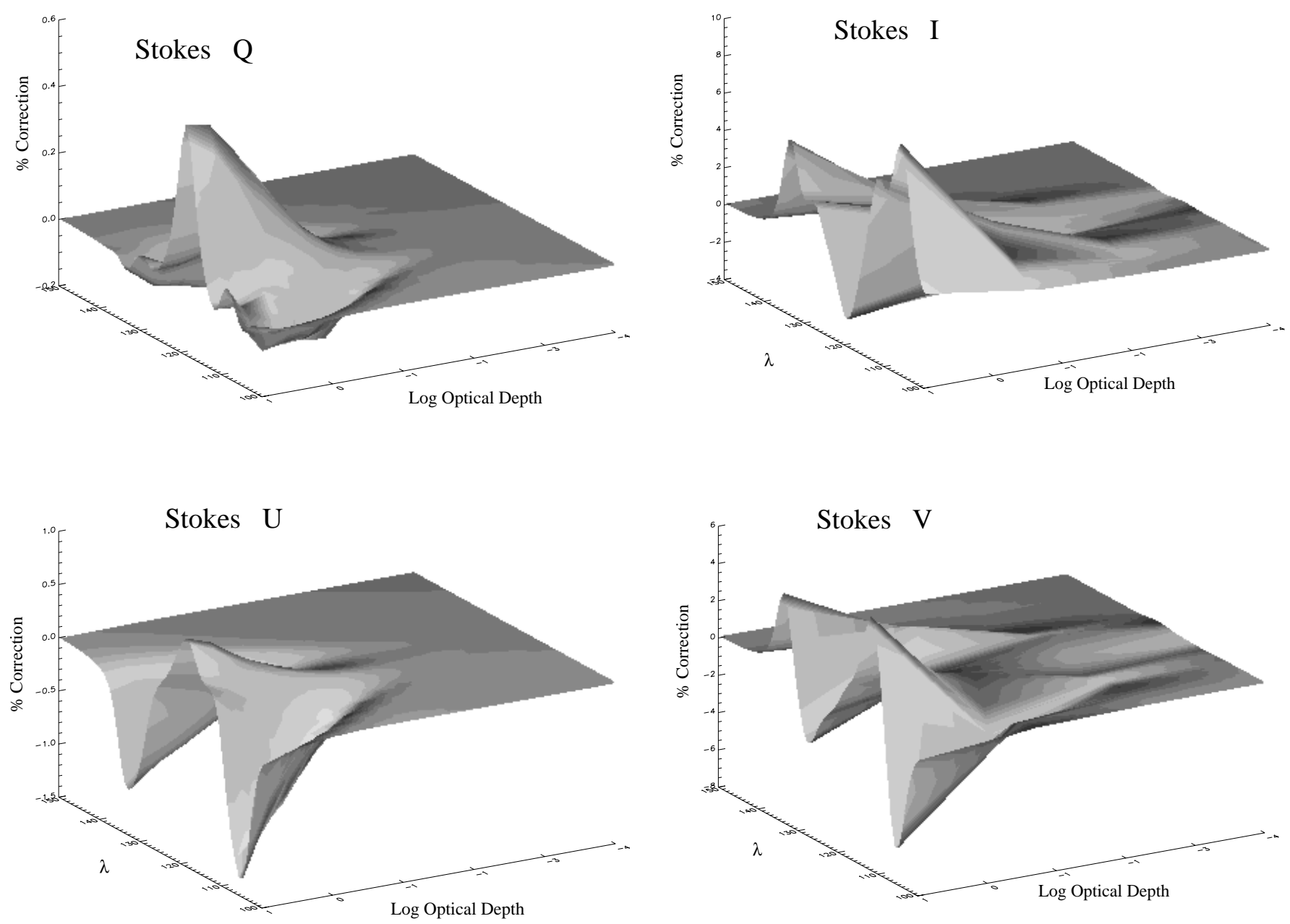

Fig. 3. Linear correction in percent of the total signal applied to $Q$ and $U$ of Fig. 1 with 15 integration layers

smaller and smaller as we approach the surface: the layers are distributed logarithmically and they are thinner near $\tau=0$. The thinner the layer the better is the approximation of a commuting matrix for it. It is also interesting to note the presence of ripples in the correction surface for Stokes $I$ and $V$. They are due to the oscillations in the evolution operator. These oscillations are one of the hardest problems for any integrator based on a sampling of the solution. If the sampling is not adequated to the frequency of oscillation an error appears. This problem has been discussed at length by Bellot Rubio et al. (1998). Any analytical solution overrides this problem, but the residual represented in Figs. 2 and 3 is based on a 2-point sampling (the linear approximation), and hence the problem arises.

The number of layers used in the previously referred figures is higher than the one usually used with DIAGONAL. Fewer layers mean thicker layers so that the correction becomes larger. We present in Figs. 4 and 5 the residuals for the same model atmosphere of the previous example integrated this time with just 3

Fig. 4. Linear correction in percent of the total signal applied to $I$ and $V$ of Fig. 1 with 3 integration layers

layers. Although the corrections are bigger, the linear approximation continues to be completely satisfactory even in these extreme cases.

\subsection{Calculation of the exponential}

Surprisingly most algorithms fail to solve this calculation, quite anodyne in the scalar case. Once more the extrapolation from the scalar case is not evident. The exponential of a matrix is defined as

$\mathrm{e}^{\Omega}=\mathbb{1}+\Omega+\frac{1}{2} \Omega^{2}+\frac{1}{3 !} \Omega^{3}+\ldots$

and it is in general not easy to calculate the entire series. Although convergence can be ensured, the series is not a power expansion. Only the presence of the growing factorial in the denominator provides a hint on how many terms may suffice to attain a given precision, and in any case the calculation is too slow. The different numerical methods used for the integration of the RTE stop the series at different points: Runge-Kutta method (Landi Degl'Innocenti 1976) stops at the 4 th order, DELO at the 2 nd order (Rees 

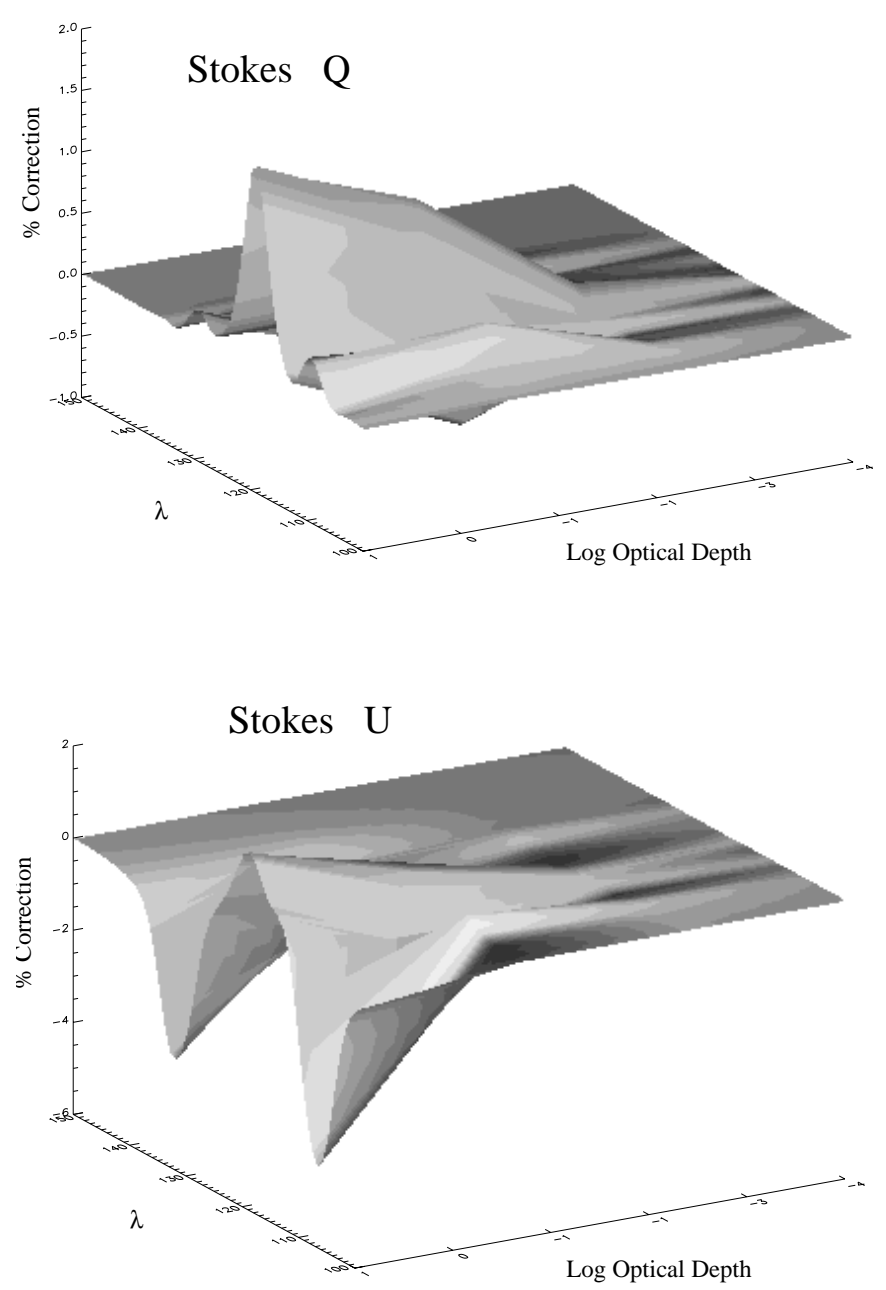

Fig. 5. Linear correction in percent on the total signal applied to $Q$ and $U$ of Fig. 1 with 3 integration layers

et al. 1989) and the Canarias Hermite method attain the 4 th order too. In view of the convergence rates of all these methods, it shall be not too far from the truth to say that the calculation of the exponential is the first source of errors in all those methods (instability problems aside).

In fact, at the cost of some more algebra, other efficient methods can be used to calculate the exponential of a matrix. We will mention here two, perhaps the most evident. The first one is to calculate the exponential of the associated diagonal matrix: if $\lambda_{i}$ are the eigenvalues of $\Omega$, and $\mathrm{T}$ the diagonalization matrix, then

$\mathrm{e}^{\Omega}=\mathrm{T}\left(\begin{array}{cccc}\mathrm{e}^{\lambda_{1}} & 0 & 0 & 0 \\ 0 & \mathrm{e}^{\lambda_{2}} & 0 & 0 \\ 0 & 0 & \mathrm{e}^{\lambda_{3}} & 0 \\ 0 & 0 & 0 & \mathrm{e}^{\lambda_{4}}\end{array}\right) \mathrm{T}^{-1}$.

This calculation requires knowledge of the eigenvalues and of the eigenvectors (to calculate $\mathrm{T}$ ). It is a very interesting method whenever we deal with an analytical matrix, whose expression is known in advance. Analytical values for the eigenvalues and eigenvectors can be calculated once for all, and the numerical code just works out the partic- ular case. This is the actual method used in DIAGONAL: the formal absorption matrix is well-known and indeed its eigenvalues and eigenvectors have already been calculated in Paper I. Hence, the exponential is calculated exactly by DIAGONAL at the price of one matrix multiplication (this interesting particularity of DIAGONAL is the origin of its name).

A second method goes through the Hamilton-Cayley theorem particularized to the exponential function. It is known from linear algebra that any matrix satisfies its characteristic polynomial. For a $4 \times 4$ matrix this is a 4 th order polynomial equation:

$a \Omega^{4}+b \Omega^{3}+c \Omega^{2}+d \Omega+e=0$.

We can write $\Omega^{4}$ as a function of the other powers of the matrix, and do it for any power higher than 4 . The exponential series in (5) becomes a polynomial of order 3 . The correct values of the coefficients of this polynomial are given by the Hamilton-Cayley theorem. This method is particularly advised for numerical matrices for which the diagonal method cannot be efficiently employed.

\subsection{Integration of the emission vector}

The integration of the inhomogeneous part of the transfer equation can be addressed by two main methods. The first one is to continue with the sampling technique as the Canarias Hermite method does. We have discussed it in the subsection on the calculation of the exponent. The second one is to give an analytical expression for the emission vector. For instance, we can assume it is linear with optical depth, as in the Milne-Eddington model atmosphere. Once we have an analytical function we can integrate the whole analytically or use numerical integration techniques. In the case of DIAGONAL we have chosen the analytical integration and used for all the tests a Milne-Eddington linear source function. This is a choice made for those tests. Other functions can be used without changing the characteristics of the code, although it is worthy to note that the analytical integration becomes rapidly very difficult due to the presence of an exponential of non-linear functions. In any case, errors do not accumulate due to this integration in the actually used code.

\subsection{Summary of errors}

As a conclusion of this section, we can summarize the sources of errors in the DIAGONAL code by saying that at worst it will behave like DELO, i.e., like a 2nd order integration technique. DIAGONAL cannot be qualified as an nth order technique in general. The reason is its mixed character. If it is asked to integrate a commuting absorption matrix it will provide an analytical result. In the worst case, when the residual becomes too big, DIAGONAL will behave as DELO. Nevertheless, this last extreme has never been found in the actual tests. 


\section{Implementation of the algorithm}

To implement DIAGONAL, we have decided to proceed in several phases. In this paper we will describe the first of them, and we will postpone the others for the near future. In the code used for comparisons with DELO we have included what we consider to be the three main characteristics of the proposed algorithm:

- The use of a diagonalization procedure to calculate the exponential in the formal solution (1).

- The possibility of treating gradients with depth for azimuth of the magnetic field.

- The linear approximation for the residual correction.

At a later stage, gradients with depth for the so-called $\alpha$ and $\xi$ angles will be treated analytically, and the analytical integration of the function $f(z)$ may be performed (the reader is once more referred to Paper I for proper definitions of these quantities and their roles in the proposed algorithm). These later developments are not expected to bring great advances or new ideas, but just an improvement over what has yet been done.

\subsection{Model atmospheres and spectral lines}

The model atmosphere used has been limited to an optical depth range of $-4.0<\log \tau<1.0$, with a grid for the integration layers distributed linearly in $\log \tau$. Optical depth, $\tau$, at $5000 \AA$ is used at any moment as integration variable. Although several spectral lines have been tested (indeed Fe I lines at $6302.5 \AA, 6301.5 \AA, 5225 \AA$ and $5250 \AA$ ), comparisons with DELO have been made for a virtual line at $5000 \AA$ and an electric dipole transition $7 D 1 \rightarrow 5 D 0$ (like the one for the Fe I line at $5225 \AA$ ). Line profiles are calculated in a wavelength range of 300 bins around central wavelength, each bin sampling $2 \mathrm{~mA}$.

We have considered not necessary for comparison purposes to use temperature and pressure parameters for description of the model atmosphere. Instead we have preferred to use more direct (in the sense of radiative transfer) parameters as Doppler width, line to continuum absorption coefficients ratio $\eta_{0}$, damping parameter $a$ and a source function linear with optical depth. From these five parameters, all are kept constant with depth, except for the source function. Constancy or variability of these parameters add nothing to comparisons with DELO, as both codes handle them numerically in the same way. However it is important to mention that DIAGONAL may in the future incorporate analytical treatment of a linear variation with depth of $\eta_{0}$, once the integration of $f(z)$ (see above) is implemented. Note that since Doppler width is a model-given parameter, microturbulence velocities are implicitly used. For the case of macroturbulence, its inclusion or exclusion in the set of model parameters will not change the results of comparisons. For the sake of simplicity, it is not used.
To these 5 parameters, one needs to add those ones related to the description of the magnetic field and the velocity in the l.o.s up to a total of 13 parameters. To describe magnetic field we use its intensity $B$, the inclination with the l.o.s $\gamma$ and the azimuth $\xi$. The first two vary linearly with $\log \tau$ in our model atmospheres, while azimuth varies linearly with optical depth $\tau$, thus allowing analytical treatment. Finally, the velocity in the l.o.s is also assumed to vary linearly with $\log \tau$.

\section{Numerical comparisons of DIAGONAL and DELO}

Currently, there exists no analytical solution for a general case of radiative transfer through a magnetized atmosphere. To prove a new numerical method involves therefore two steps. First, to ensure that at least for the simplest cases, for which an analytical solution is known, the correct solution is obtained. These cases are almost reduced to the constant absorption matrix case, for which, as Bellot Rubio et al. (1998) show, any numerical method better than second order gives the proper solution. In the second step, for the general case, the new method must be compared to a previous well-tested numerical method, in search of convergence to the very same solution for both methods. This section is devoted to these comparisons. In the role of a previous well tested numerical method we have chosen DELO (Rees et al. 1989). DELO is known to approach asymptotically the solution for any model atmosphere. We will therefore consider its solution obtained with a big number of layers (usually around 500 layers suffice) as reference solution. Indeed, DIAGONAL is shown to present the same behaviour, and needs fewer layers to converge: Usually 100 layers will suffice, keeping integration errors under computer precision.

Before starting with the comparisons, we note that for very specific models (namely those characterized by a constant magnetic field intensity and inclination, and a constant velocity field in the l.o.s. but an azimuth of magnetic field varying linearly with depth) DIAGONAL provides an exact solution, independently of the number of layers. We can take advantage of that to test the convergence rate of DELO in a non-trivial case (if by trivial we refer to the Rachkowsky solution). The model presented in Table 1 has been used to this purpose. Azimuth gradient is bigger than the usual values considered for solar model atmospheres, but we wanted to observe the convergence rate, and not absolute values of precision. We have integrated the model with DELO using 30 to 530 integration layers. DIAGONAL provided the correct solution and the precision of DELO's integration is referred to this solution. The results are shown in Fig. 6.

DELO is shown to be a second-order approximation while solving for Milne-Eddington models (although in this particular case, a null second derivative of the source 
Table 1. Model atmosphere with non-zero azimuth gradient used to test DELO's quadratic convergence.

$\begin{array}{lr}\text { Doppler width } & 29.4 \AA \\ \text { Line to continuum ratio } & 10 \\ \text { Damping parameter } & 0.001 \\ \text { Source function at } \tau=0 & 1 \\ \text { Source function gradient } & 2 \\ \text { Magnetic field intensity } & 1500 \mathrm{G} \\ \text { Magnetic field gradient } & 0 \\ \text { Azimuth at } \tau=0 & 45^{\circ} \\ \text { Azimuth gradient } & 90^{\circ} \\ \text { Inclination } & 50^{\circ} \\ \text { Inclination gradient } & 0^{\circ} \\ \text { Velocity at } \tau=0\left(\mathrm{~km} \mathrm{~s}^{-1}\right) & 0 \\ \text { Velocity gradient }\left(\mathrm{km} \mathrm{s}^{-1}\right) & 0\end{array}$
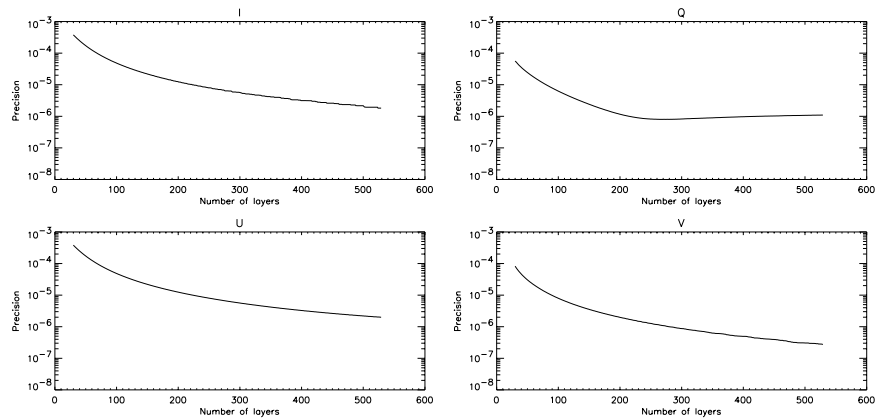

Fig. 6. Precision versus number of layers figure for DELO with the model atmosphere presented in Table 1

function makes exact any approximation to second or bigger order). Bellot Rubio et al. showed that DELO converged also as a second-order approximation for a general atmosphere solved numerically. The results shown in Fig. 6 confirm this statement for a non-trivial analytical solution: DELO converges quadratically with the inverse of the thickness of the integration layer.

Next, we present the two models which have served to verify the convergence of DIAGONAL. We have chosen first a model with a gradient of magnetic field intensity as the sole varying parameter, and a second one in which gradients of azimuth and l.o.s. velocity are also considered. Table 2 describes both models (labeled Model 1 and Model 2 ). By selecting these two particular models we want to characterize the behaviour of DIAGONAL. As stated in previous sections, it can be summarized in 3 basic points:

1. Analytic solution for models with linear variation of azimuth.

2. Exact calculation of the exponential.

3. Linear approximation used for the residual correction.

Model 1 has constant with depth parameters except for magnetic intensity. In terms of solutions DIAGONAL is not better than DELO. In fact the comparison graphic (Fig. 7) shows that the convergence rate is equal for both codes from a certain number of layers. The difference is the exact calculation of the exponential, which improves the
Table 2. Model atmospheres for comparisons

\begin{tabular}{l|rr} 
& Model 1 & Model 2 \\
\hline Doppler width & $29.4 \AA$ & $29.4 \AA$ \\
Line to continuum ratio & 10 & 10 \\
Damping parameter & 0.001 & 0.001 \\
Source function at $\tau=0$ & 1 & 1 \\
Source function gradient & 2 & 2 \\
Magnetic field intensity at $\tau=0$ & $1500 \mathrm{G}$ & $1500 \mathrm{G}$ \\
Magnetic field gradient & $1300 \mathrm{G} / \tau$ & $300 \mathrm{G} / \tau$ \\
Azimuth at $\tau=0$ & $45^{\circ}$ & $47^{\circ}$ \\
Azimuth gradient & 0 & $45^{\circ} / \tau$ \\
Inclination & $50^{\circ}$ & $50^{\circ}$ \\
Inclination gradient & $0^{\circ}$ & $0^{\circ}$ \\
Velocity at $\tau=0\left(\mathrm{~km} \mathrm{~s}^{-1}\right)$ & 0 & 0 \\
Velocity gradient $\left(\mathrm{km} \mathrm{s}^{-1}\right)$ & 0 & 0.4
\end{tabular}
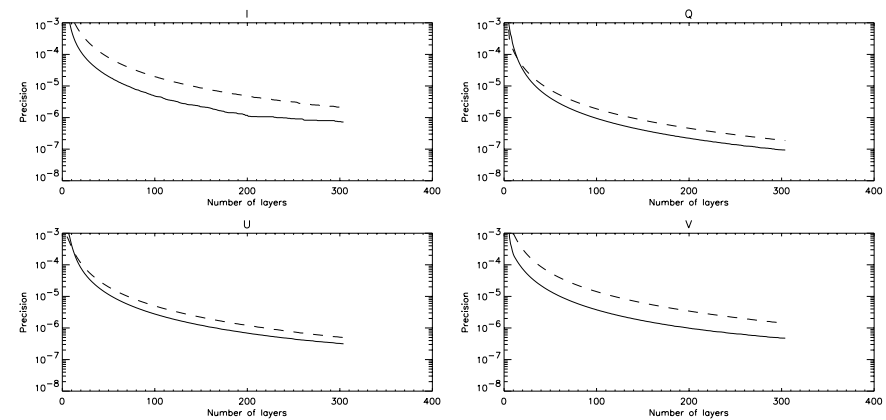

Fig. 7. Precision versus number of layers figure for DELO (dashed line) and DIAGONAL (continuous line) with model atmosphere 1
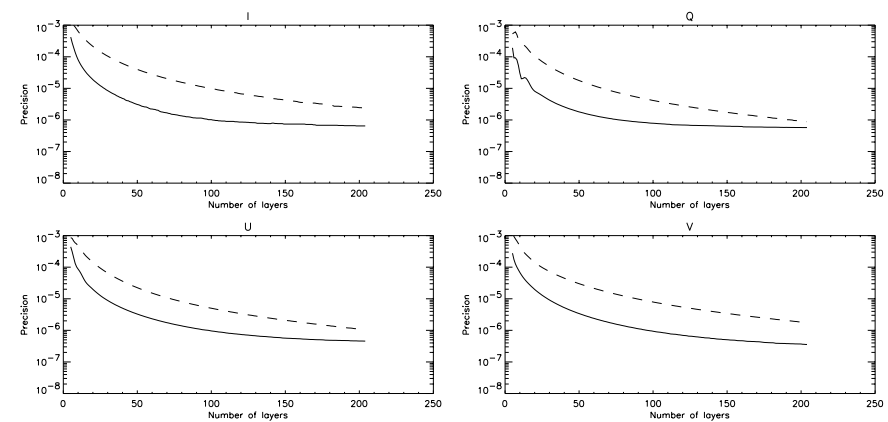

Fig. 8. Precision versus number of layers figure for DELO (dashed line) and DIAGONAL (continuous line) with model atmosphere 2

convergence of DIAGONAL for a small number of layers. The bigger the number of layers, the better the DELO's second order approximation for the exponential is. Once DELO compensates for the advantage of DIAGONAL, both codes behave similarly. This paragraph can be repeated for Model 2 (Fig. 8). But in this case DIAGONAL treats analytically the non-zero azimuth gradient, thus increasing its advantage over DELO, specially when few integration layers are used (see the referred figure). 


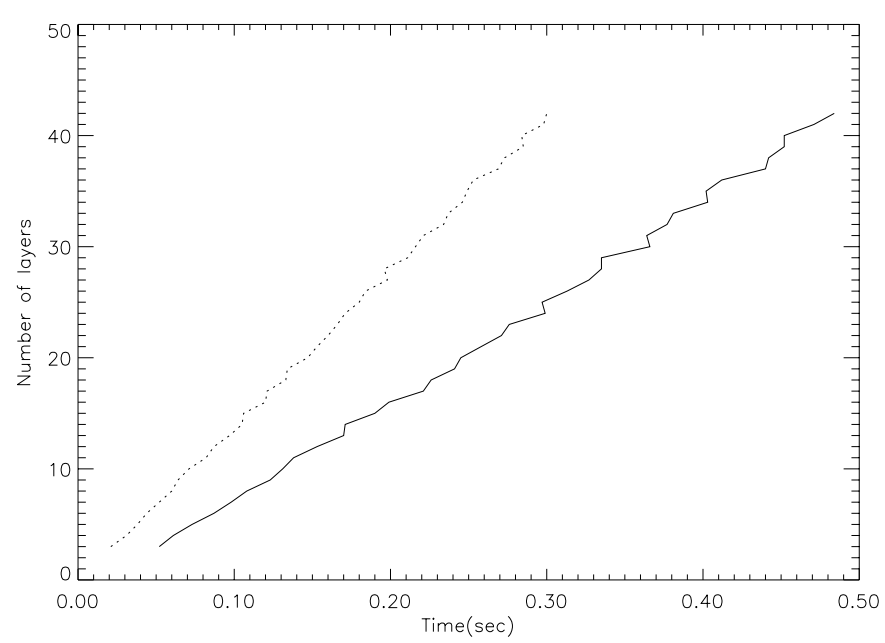

Fig. 9. Comparison of time versus number of layers for both: DELO (points line) and DIAGONAL (continuous line)

After these 2 examples, the following statement appears to be a reasonable description of DIAGONAL: This new code behaves at worst as DELO, but always presents a faster convergence for a small number of layers. The first part of this assertion was already predicted in our error analysis in Sect. 2, the second part was the searched objective for DIAGONAL, and this fast convergence shows the success of the code. It means that the analytical approach is indeed very useful and desirable. The elaborated algebra implicit in the DIAGONAL code implies a longer integration time per layer than DELO. Therefore, it is in the interest of this new code to use as few layers as possible to attain a desired precision. To illustrate this point of DIAGONAL, namely its slowness in integrating a single layer, we present in Fig. 9 a graph of time versus number of layers for both DELO and DIAGONAL. DELO appears to be 1.6 times faster per layer than DIAGONAL. If DIAGONAL is still a faster code, it is due to the fact that it can attain the desired precision with up to 6 times fewer layers than DELO.

\section{Conclusions}

This paper represents the actual realization of the ideas presented in Paper I. The performance of the new numerical code is a clear indication that analytical studies on the RTE, as the one presented in Paper I, are of great importance not only from the theoretical point of view, but also from the practical point of view, as they can provide us with high-performance codes capable of solving more and more complicated model atmospheres in reasonable times.

Measurement of magnetic fields in the sun rely on inversion codes based nowadays on minimization techniques which make an intensive use of integrators. Any improvement in the performance of these integrators involves an immediate improvement on the results obtained with the inversors. The number of instruments providing polarimetric information is in permanent growing, as well as the quantity of information provided by each one of them. It is an imperious necessity therefore to accelerate and improve at the same time the available integration and inversion methods if we want to deal with all that fresh data.

Two main trends can be followed for that purpose, the first one is to rely on purely numerical techniques, the second one to deepen in the knowledge of the RTE to take advantage of its symmetries, characteristics and so on. DIAGONAL was conceived in this second spirit. It is worth noting that both trends may be combined and profit from a theoretical advantage by means of powerful numerical techniques. In this sense DIAGONAL will be a first prototype of new integrators grounded on deep theoretical basis. The code is available, upon request to the authors.

\section{References}

Bellot Rubio L., Ruiz Cobo B., Collados M., 1998, ApJ 506, 805

Landi Degl'Innocenti E., Landi Degl'Innocenti M., 1985, Sol. Phys. 97, 239

Landi Degl'Innocenti E., 1976, A\&AS 25, 379

Landi Degl'Innocenti E., 1987, Kalkofen W. (ed.), Numerical Radiative Transfer, pp. $265-278$

Lites B., Skumanich A., Rees D.E., Murphy G.A., 1988, Astrophysics 330, 493

López Ariste A., Semel M., 1998, Nagendra K. and Stenflo J. (eds.), Proc. 2nd Solar Polarization Workshop, Bangalore, pp. 157-169

López Ariste A., Semel M., 1999, A\&A (accepted for publication in $\mathrm{A} \& \mathrm{~A}$ )

Magnus W., 1954, Comm. Pure. App. Math. VII, 649

Rachkowsky D., 1967, Izv. Krym. Astrofiz. Obs. 37, 56

Rees D., Murphy G.A., Durrant C.J., 1989, ApJ 339, 1093

Ruiz Cobo B., Bellot Rubio L., Collados M., 1998, Nagendra K. and Stenflo J. (eds.), Proc. 2nd Solar Polarization Workshop, pp. 231-240

Semel M., López Ariste A., 1999, A\&A 342, 201 